\title{
Brazil Conference on Treatment of Leprosy
}

Abstracted and translated by Dr. J. W. Lindsay from Revista de Leprologia de Sao Paulo, June 1935.

From the 25th to the 27th of June, 1935, there was held the first conference of doctors who work in the various hospitals of the Leprosy Association of the State of Sao Paulo, Brazil, for the object of exchanging ideas upon the treatment of leprosy. There were present at the meetings Dr. E. Burnet, Director of the League of Nations' Leprosy Prophylaxis Section, and also Professor Edward Rabello, President of the Rio de Janeiro branch of the International Association of Leprosy.

At the first meeting papers were read on the use of methylene blue, the old remedy, more recently advocated by Montel, as inspiring from its results very high hopes both on the part of doctons and of patients. Montel and his fellow workers and others who hastened to experiment with the new method, drew such hasty conclusions, that others proceeding more deliberately, and with more careful examination of the results obtained by themselves, threw doubt upon the first reports, and even went so far as to condemn the method because of its very slight specific action, and because of its very evident secondary toxicity, thus disappointing the first high hopes in their general anxiety to find a specific remedy for the disease.

Some six lectures were given on the use of methylene blue at this conference, and the conclusions reached were very varied. None of the investigators, even the most optimistic, showed the same enthusiasm as Montel had done when, as proved by his marvellous results, he claimed that methylene blue is three times more active than chaulmoogra. Alongside of the very encouraging results obtained, especially the immediate results in the improvement of infiltrated lesions, cicatization of chronic ulcers, relief of neuralgias and " reactions," there have to be recorded also the late toxicity secondary to high dosage, with certain untoward reactions, asthenia, wasting and even one fatal accident. There was unanimity in recognising the affinity of methylene blue for the diseased tissue, which becomes impregnated with it at the very first injections; there was the same unanimity as to the fact that those lesions very soon get "disinfiltrated " of the remedy, recur with greater or lesser intensity, especially the tubercles and the infiltrations; but there was no unanimity 
in admitting any definitely curative therapeutic action as superior to that of chaulmoogra.

The investigation of results obtained were on the whole not very encouraging. The question of dosage was fully discussed. The original method of Montel, who prescribed very high doses of the remedy was modified always with lesser dosage. The same impregnation of the lesions with the blue resulted with the smaller dosage, and there was better tolerance. In the treatment of the "algias" and "reactions" the results were more or less encouraging, although there were various inexplicable discrepancies in the reports.

The conclusions reached were as follows:-Methylene blue has great affinity for the diseased tissue: it impregnates it and produces degeneration of the Bacillus of Hansen as proved by Markianos and Lépine : this impregnation is due to an affinity for the tissues altered by the bacillary toxins, or to the fact that the blue being a "vital " colouring agent is deposited where the endothelial reticulum has been modified by the action of the bacillus or its toxins, and their presence has an inhibitory effect with the result that secondary toxic effects are produced. There are delicate questions of biopathology which careful and patient study may solve, and we consider that the remedy ought not to be abandoned. The careful selection of cases, the patient study of the visceral reactions to associated remedies, chaulmoogra, cholesterin, lecithin or the benzylic radical, are factors that ought to guide investigators towards the best use that can be made of the therapeutic agent in the fight against Hansen's disease, for which actually there are so few available remedies.

At the second meeting six papers were read and special attention was given to the treatment of the leprotic "algias" by methylene blue, and reports read of the use of rattlesnake anti-venom and of osmic acid. It was remarked how leprosy may cause, besides the multiform skin lesions, internal lesions of the most varied kinds, with a symptomatology equally varied. Among these may be mentioned as causing the greatest suffering to the patients, the "algias" of every kind, myalgias, neuralgias, ostealgias, which are not relieved or only very uncertainly by the ordinary analgesics.

Are these "algias" of a toxic nature? Or are they simply inflammatory, the result of the presence of the bacilli disseminated through the various invasions of the disease? We ourselves do not believe that they are due to 
the irritation produced by the presence of the bacilli, having noted that the wonse tuberose cases are those that are least subject to the "algias." They are evidently of a toxic nature, the same as the "reactions," the febrile attacks and the cachexia.

It is the want of exact knowledge of their nature that explains the failure of our therapeutics, and also explains the anxiety of the leprologists to discover a remedy that will relieve the patient of the most troublesome symptom of his disease.

Among the complications in the development of leprosy, the ocular lesions, because of their frequency, and their great risk to the patients and the danger even of blindness, are very specially attended to in the different leprosaria of the State, each of which has its own eye specialist. The lesions of the eyes are very varied, some of an acute nature, result of treatment given or of the evolution of the disease, some associated with the "reactions," othens of a chronic kind of the eye itself or its appendages.

From the reports given it can be seen how very complicated is the treatment of leprosy in those hospitals, and what care is required in the carrying of it out for the good of the patients, and how on the scientific side they are quite on a par with the best known institutions of a similar character. Sao Paulo, in the opinion of Professor Burnet, is developing by itself one of the best leprological centres in the world.

Dr. Renato Braga reported on his treatment by methylene blue of 122 cases. Having found that the treatment gave the best results in advanced cases of leprosy, especially of the "tuberous" variety, the majority of his patients treated were of the kind described. While not sharing the optimism of Montel, he does not hesitate to affirm that the immediate results obtained with methylene blue are, although uncertain, really superior to those obtained with chaulmoogra and its derivatives. The favourable modifications observed in the advanced cases, especially of nodular type, in generalised infiltration and in oedemas and trophic ulcerations, were so apparent after the very first injections, that he feels he is guilty of no exaggeration in affirming that identical results could only have been obtained with a very prolonged treatment by chaulmoogra derivatives in whatever way administered, whether by local applications or intradermic injections.

Certain cases that had presented constant intolerance to chaulmoogra treatment were found to be more amenable to 
methylene blue, and benefitted by it. The general practice of this observer was that of combined treatment by methylene blue and chaulmoogra, the latter in the form of gelatin capsules of the oil given by mouth in accordance with the tolerance of the patient. The idea underlying this method was that propounded by Marchoux and Millian, viz., that the blue may serve as a real vector for other antileprotic remedies of greater activity; or it may be that with its prolonged use more favourable conditions may be produced for a better and more intense action of other remedies, just as Boinet showed that methylene blue used in malaria favours a better fixation of quinine on the plasmodia.

For the investigation of any morphological and chromatic transformations of the bacillus in specimens obtained from patients after prolonged treatment with methylene blue, thirty different slides were examined, but no such modifications were observed, as would prove direct action of the remedy upon the bacillus of Hansen.

Although uncertain as to its mode of action, this observer, judging from the good results obtained in some cases, considers it justifiable to continue the use of the remedy, as well as to continue the study of the effects of other colouring agents in the hope of discovering some remedy more active and more constant in its results than chaulmoogra or its derivatives.

Dr. Flavio Maurano, of the Sanatorium Padre Bento, reported on 44 patients treated with methylene blue, and gave as his conclusions that:-

1. The intravenous injection of methylene blue in leprosy, according to the technique of Montel, did not prove of any efficacy, while there was noted evidence of toxicity.

2. In $25 \%$ of the cases observed by us, there was aggravation of the skin lesions, and in $18 \%$ of the general condition of the patients. The others remained indifferent to the treatment, with the exception of one case of the macular type in which the lesions disappeared.

3. In regard to the lepra reaction, in one case it disappeared, in $12 \%$ there was exaggeration, and in $10 \%$ attenuation.

In a series of 51 cases treated with methylene blue by Dr. J. Correa de Carvalho, of Colonia Aymores, the results were varied, but the conclusion arrived at was that :-

"although it may be that methylene blue cannot guarantee positive cures, nor challenge the supremacy of chaulmoogra oil in the treatment of leprosy, it ought, nevertheless, to figure in the therapeutic arsenal against the disease as an auxiliary in the great Leprosaria where the cases of nodular leprosy are numerous." 
Drs. Edison Costa Valente and E. Luiz Marino Bechelli, of Colonia Cocaes, reported on the use of methylene blue in 37 cases of leprotic reaction, and its effect upon the cutaneous manifestations. It was found that the cases that were cured of leprotic reaction, or the ones that got relief were those treated with the higher doses of the remedy, from 25 c.c., up to 40 c.c., of $1 \%$ solution of Montel. The weaker doses, 15 c.c., to 25 c.c., did not have the desired effect, although in the whole series of cases about one-third of them had favourable results.

In the treatment of leprous " algias " by methylene blue, Dr. Luiz Marino Bechelli, of Colonia Cocaes, made observations of fifteen cases and reported that:-

1. Methylene blue is a valuable remedy in the therapeutics of leprous algias-in only one out of the 15 cases did it fail.

2. The best results obtained were with weaker doses of the solution, higher doses only being used when the first produced no effect.

3. Only a few patients suffered from complications such as loss of appetite, giddiness, salivation or rigors; some had reappearance of leprotic reaction, whereas others were greatly improved or cured of their cutaneous eruption.

Dr. Francisco Ribeiro Arantes, of Sanatorio Padre Bento, reported on "Accidents Observed during the Treatment of Leprosy with Methylene Blue by the method of Montel," with the following conclusions:-

1. Methylene blue has a beneficial effect upon acute febrile attacks in a small number of cases, its effect is temporary, and it is inferior in effect to other remedies used in similar cases (calcium, tartar E., hyposulphite, triplaflavin, fuadin, etc.).

2. Methylene blue has a more certain effect upon the pains or "algias" and especially those of "neuritis" in half of the cases treated by us (10) of leprotic reaction and pains; the pains were relieved and in some cases completely disappeared. In the cases where there was improvement with diminution of the pains, the acute attacks recurred at longer intervals and with less intensity. We are of opinion that smaller doses should be employed in these circumstances.

3. Methylene blue may cause the appearance of lepra reaction with or without pains in patients in whom up to then such complications had not been observed.

4. Methylene blue is not an innocuous drug and may give rise to slighter or graver phenomena of intoxication.

5. The complications of "toxic hepatitis" observed by us were in " mixed" cases, in which there was a "tuberous" element.

6. In these cases of "hepatitis" it was noted that there was no elimination of the blue in the faeces, which proves that it had been retained in the affected cells of the liver.

7. In the cases of proper tolerance, after proper dosage is reached, 
the blue is eliminated in the bile, the faeces being coloured blue, with or without diarrhœa.

8. The sedimentation index showed notable variation.

9. The patient's weight showed variations in accordance with the accidents observed.

10. Methylene blue may cause death by atrophy of the liver.

11. Phenomena of intoxication are always apparent in the digestive apparatus, the liver being the first to show signs, and later the kidney, which are attacked after the appearance of the hepatic lesion.

12. Methylene blue by Montel's dosage is toxic and dangerous.

Dr. Joao de Moraes Junion, of Colonia Piripitinguy, reported on the treatment of the "algias" in leprosy patients, with special reference to the use of snake antivenom. After giving a historical resumé of the treatment from the first reported cases of sudden complete cure of leprosy after rattlesnake bite, the observer refers to later experiments in different parts of the world. Of very special interest were the reported cures from the anti-venoms, prepared in the Butantan Institute of Brazil, and communicated by Vital Brazil.

The author had a series of 30 cases of "algias" of which seven would not yield to the other commoner treatments; these seven were used for the experiments. After an interval of eight days without any treatment, daily injections were begun of anti-venom, using the route advised by other experimenters, viz., the subcutaneous, and trying always as far as possible to inject the anti-venom into the painful part, or its neighbourhood. Two injections were given daily.

The results obtained were very encouraging. With the exception of one case the results were good. In the other six, 8 injections were sufficient to produce complete relief that lasted from 45 to 90 days. In one case the pains reappeared after 30 days of the beginning of the treatment, and never completely disappeared. In spite of this the author holds that the action of the anti-venom is more or less persistent, since, even in cases of recurrence of the pains, they have never been of the original intensity. No complications or disturbances were observed as results of the injections, neither general nor febrile reaction nor any accident of any kind. The use of anti-venoms has one drawback and it is that the continued use of them immunise the patients against any good effects of the same anti-venoms upon the leprous lesions, but this difficulty may be overcome by changing the type of anti-venom, using alternately the anti-venoms of different reptiles. 
The latest contribution to the study of the effect of antivenom upon leprosy "algias" is that of G. de La Plaza, M. Vegas and B. Gomez, in the Revista de la Poloclinica Caracas, Venezuela, April, 1935, who, writing of the use of the "Neurotoxin of Rattle Snakes," say:-

"The neurotoxin was used in 30 cases of "algias" in leprosy, with the following results: Cured 14 cases; improved 14 cases; failures 2 cases. The neurotoxin of rattlesnake in doses of $1 / 10$ milligram exercises a markedly sedative effect upon the "algias" of leprosy, chiefly in the nerve or mixed varieties, and particularly in cases of "arthralgias" these painful complications of leprosy have been so rebellious to other therapeutic measures, that we consider the results obtained by us as very encouraging."

"Osmic Acid Intravenously for Acute Leprous Neuritis." Dr. Argemiro Rodrigues de Souza, of the Colonia Rodrigues, reported as having been perhaps the first to use intravenous injections of 2 per mille osmic acid for the treatment of the acute neuritis complication of leprosy. Series of 5 to 10 injections were given of 2 c.c., on alternate days.

The treatment was employed in 18 cases and with very remarkable results. There were no signs of intolerance in any of the cases, nor any disturbances. Neuben had given warning of cases of nephritis and diarrhœas, but the author had no such experience with his cases. The later results of intravenous osmic acid injections of 2 per mille in the 18 cases observed were always good, there being complete disappearance of the pains under the treatment.

"Thyroid Gland Disturbance in Leprosy, and their Treatment " were reported on by Dr. Argemiro Rodrigues de Souza, of Colony Pirapitinguy. . In the case described, one of " mixed " leprosy, with maculae and erythemes, there were evident signs of hypo-thryoidism, obesity, mental apathy, and oedemas. Tablets of Thyroid Extract 0.30 grs., 3 times a day were prescribed and the effect was very remarkable. The oedemas rapidly subsided and the erythemas disappeared, and by the end of two months the patient had completely recovered from the symptoms described of hypo-thyroidism which had been the complication that had hindered any beneficial effects that might have been got from the specific treatment of the leprous condition.

Dr. J. Mendonca Barros, of the Sanatorio Padre Bento, reported on their treatment of ocular complications of leprosy. In acute cases of ocular lesions their method was :-

1. The suspension of all specific treatment, (chaulmoogra, etc.). 
2. General desensitising treatment, especially auto-hœmotherapy.

3. Special symptomatic treatment as required.

The hyperplastic forms of keratitis, as well as the episcleroconjunctival lepromas were treated by excision and cauterization.

In a series of 16 patients treated with Solganol B., intramuscularly the results were not encouraging, only three of the cases showing any improvement.

"The Treatment of Ocular Complications in Leprosy." Dr. Francisco Amendola, of the Colonia Santa Angelo, reported on the results obtained in their hospital in the treatment of acute forms of eye complications, and in the surgical treatment of chronic forms. The conclusions arrived at were the following:-

1. The treatment of acute forms of eye disease is eclectic, depending on the general condition of the patient.

2. The hyperplastic lesions of the cornea ought to be operated on, as their development marks a pause in the process of the ocular trouble.

3. Iredectomy in ocular affection of leprosy is only indicated in cases where the iris itself is not markedly affected.

4. Canthorrhapy gives satisfactory results in cases of orbicular paralysis, especially when corneal lesions have been caused by permanent exposure of the cornea.

"Valuation of the Results of Treatment at the Sanatorium Padre Bento" recorded by Dr. Lauro de Souza Lima.

1. The actual results of special anti-leprotic treatment appear to be conditional upon a combination of circumstances, the chief of which is that of the general treatment of the case. It is considered advisable to institute such treatment systematically in all cases, as the general low condition of the patients may be as chronic as is the disease they suffer from.

2. We are not in a position to give a definite opinion upon the permanency of the results obtained, favourable or otherwise, because of the insufficiency of the observation period, and because a great number of the patients escape from our control with their transference to dispensary treatment.

3. In order that the Department of Leprosy Prophylaxis may be able in the future to pronounce upon the efficacy of antileprotic treatment, based upon the abundant material at its disposal, it would be convenient:

(a) to perfect the clinical classification of the cases under treatment, so that it may be possible to indicate exactly the precise gravity of the disease, eliminating as far as possible the personal coefficient of the doctor, a thing which is not easy with the classifications at present in use, and without which statistics are liable to be deceptive.

(b) to tabulate the methods of verification of progress attained, and the opinions of a valuation of the results by the 
exact delimitation of what ought to be understood by the term " much improved," " unaltered," etc., as well as of the number of bacterioscopic examinations necessary.

(c) to tabulate, as far as possible, all treatment given.

"Routine Treatment of Leprosy in the Sanatorium Padre Bento " as described by Dr. Lauro Souza Lima. The chief principles followed are :-

1. The complete intelligent co-operation of the patient must be secured.

2. The uselessness must be admitted of treatment in cases in which there remain only the stigmas of a disease that has disappeared, whether in advanced cases or in the initial period of new cases. Such ought to be separated from other cases undergoing specific treatment.

3. The aim must be to raise the general condition and resistance of the patient to the highest level possible.

These principles must be followed in conjunction with all therapeutic measures of general treatment. As practical results obtained from the following of these principles, the following suggestions are made :-

1. To secure the intensification by all means within our reach, of the social life of the hospitals.

2. To secure the transference to a special hospital of cases that have remained stationary in the later stages of the disease, in order to avoid that their presence among " treatable" patients may not discredit the treatment.

3. To secure the transference for dispensary treatment of adult patients that have remained stationary in the initial period, but only for purposes of observation.

4. To secure the preventive transference of child patients that have remained stationary in the initial period for observation until puberty.

"Intradermic Infiltration, or 'Plancha' Method, was described by Dr. Lauro de Souza Lima, of the Sanatorio Padre Bento.

"Experiments in the Treatment of Leprosy by Autohœmotherapy " were described by Dr. José Correa de Carvalho, of the Colonia Aymores. Of 15 cases treated by auto-hœmotherapy, 5 had their condition aggravated, while 10 showed considerable improvement. The treatment was considered worth trying in febrile eruptions. 